\title{
Ensino clínico na enfermagem: a trajetória da produção científica
}

\author{
Clinical teaching in nursing: the scientific production path
}

Enseñanza clínica en la enfermería: la trayectoria de la producción científica

\section{Glauteice Freitas Guedes', Conceição Vieira da Silva Ohara', Gilberto Tadeu Reis da Silva", Glaci Regina Rodrigues de Melo Franco'}

'Universidade Federal de São Paulo. Departamento de Enfermagem. Escola Paulista de Enfermagem. São Paulo, SP Faculdade Santa Marcelina. Grupo de Pesquisa de Formação em Educação em Saúde. São Paulo, SP

Submissão: $03 / 04 / 2008$

Aprovação: 16/02/2009

\section{RESUMO}

Trata-se de um estudo bibliográfico, no qual se realizou um levantamento da produção científica de enfermagem referente ao ensino clínico no Catálogo de Dissertações e Teses do Centro de Estudos e Pesquisas em Enfermagem (CEPEn) da Associação Brasileira de Enfermagem - ABEn, no período de 1979 a 2004. Objetivou descrever a distribuição desses estudos Quanto ao número de publicações, título defendido, origem da publicação, ano de defesa, metodologia utilizada e temática abordada. Concluiu-se Que a produção científica nessa área ainda está incipiente. Assim, poderemos aperfeiçoar nossos fazeres enQuanto educadores e ocupar de forma competente e Qualificada nosso espaço no sistema de educação e saúde.

Descritores: Enfermagem; Ensino; Educação.

\section{ABSTRACT}

It's a bibliographic study, in which it was made a scientific production survey on nursing referring to clinical teaching in the Catálogo de Dissertações e Teses do Centro de Estudos e Pesquisas em Enfermagem (CEPEn) (Nursing Study and Research Center's Dissertation and Thesis Catalog) from Associação Brasileira de Enfermagem - ABEn (Brazilian Nursing Association) between 1979 and 2004. was aimed to describe the study distribution regarding to the number of publishing, applied title, publishing origin, year of application, methodology used and the theme approached. It was concluded that scientific production in this area is still incipient. Thus, we can improve our duties as an educator and occupy our space in the education and health in a competent and Qualified way.

Descriptors: Nursing; Teaching; Education.

\section{RESUMEN}

Tratase de un estudio bibliográfico, en lo cual se realizó un estudio de la producción científica de enfermería referente a la enseñanza clínica en el Catálogo de Disertaciones y Tesis del Centro de Estudios e Investigación en Enfermería (CEPEn) de la Asociación Brasileña de Enfermería - ABEn en el período de 1979 a 2004. Su objetivo fue describir la distribución de esos estudios cuanto al número de publicaciones, título defendido, origen de la publicación, año de defensa, metodología utilizada y temática abordada. Se concluyó Que la producción científica en esa área aún está incipiente. Así, podremos perfeccionar nuestras tareas como educador y ocupar de forma competente y calificada su espacio en el sistema de educación y salud.

Descriptores: Enfermería; Enseñanza; Educación. 


\section{INTRODUÇÃO}

Ao pensarmos em ensino no campo da enfermagem, emerge uma Questão muito importante relacionada ao seu processo: o ensino clínico nos campos da prática, seja no hospital, na comunidade, nas escolas ou em unidades básicas de saúde. O trabalho da enfermagem inclui atividades de naturezas propedêutica e terapêutica específicas, o Que demanda a necessidade de profissionais com níveis diferenciados de formação. Nesse contexto, o enfermeiro é um profissional Que, durante sua formação, deve ter obrigatoriamente atividades de ensino teórico e prático, as Quais se darão nos campos clínicos ou campos de estágio.

O ensino clínico na enfermagem é usualmente designado nas instituições como estágio e proporciona aos acadêmicos momentos de aproximação com a vida profissional( ${ }^{(1)}$. E pode ser definido como uma etapa de aplicação do conhecimento teórico Que pode gerar reflexão crítica e o aperfeiçoamento de habilidades em situações reais e proporciona ao aluno unir o saber com o fazer, e, se bem direcionado, levará o discente a desenvolver um agir mais consciente, crítico e criativo $^{(2,3)}$

O estudo teórico no campo da enfermagem deve emanar dos problemas práticos vivenciados pelos alunos na sua prática de aprendizagem, assim, a proposta do Currículo de Enfermagem prevê Que a formação do enfermeiro contemple atividades práticas e teóricas ${ }^{(4)}$. Isso significa Que além das atividades teóricas como aulas, seminários e outros estudos da mesma natureza, o currículo deverá abordar o ensino prático comumente adotado pelas escolas como os laboratórios, ensino clínico nas diversas áreas da assistência e dos serviços de saúde hospitalares e também das unidades básicas de saúde.

O cuidado de enfermagem precisa ser aprendido mediante o contato direto do aluno com o paciente. Dessa forma o estágio ou o ensino clínico aparece como parte integrante dos currículos na formação do enfermeiro. Essa atividade proporciona ao discente diferentes contextos na prática e exerce uma influência importante na formação dos enfermeiros ${ }^{(5)}$, pois a presença do docente e de enfermeiros envolvidos no ensino teórico-prático promove a segurança dos alunos e colabora para estabelecer as relações nos diferentes contextos da prática ${ }^{(6)}$.

$\mathrm{Na}$ área da saúde, o estágio é uma atividade obrigatória prevista nos currículos de graduação, regulamentada pela Lei 6494/77 e pelo decreto 8797/82 do Ministério da Educação e é entendido como uma atividade de aprendizagem social, profissional e cultural proporcionada ao estudante pela participação em situações reais de vida e trabalho de seu meio, sendo realizado na comunidade em geral ou junto a pessoas jurídicas de direito público ou privado sob responsabilidade e coordenação da instituição de ensino.

Com os estudos citados, percebemos a obrigatoriedade e a importância do ensino clínico na formação do enfermeiro, o Que reforça ainda mais a necessidade da construção de conhecimentos e da busca de novas descobertas Que abrangem aspectos da temática em Questão.

Considerando Que a prática da assistência de enfermagem é uma atividade essencial para a formação profissional do enfermeiro, pois é por meio dessa experiência Que o enfermeiro constrói seu conhecimento, julgou-se pertinente a realização de um estudo Que pudesse demonstrar a real produção do conhecimento sobre a temática aQui abordada, Que poderá subsidiar o desenvolvimento de pesquisas sobre o tema ensino clínico, além de identificar aspectos Que estão relacionados ao processo de ensino-aprendizagem no campo da enfermagem.

Sendo assim, buscando aproximações para contextualizar o ensino clínico na área de enfermagem, por meio de referenciais teóricos Que estão sendo utilizados, procurou-se levantar a produção científica de enfermagem contida no Catálogo de Dissertações e Teses do Centro de Estudos e Pesquisas em Enfermagem (CEPEn) ${ }^{(7)}$ da Associação Brasileira de Enfermagem (ABEn) no período de 1979 a 2004.

\section{OBJETIVOS}

- Conhecer a produção científica de dissertações e teses que abordem ensino clínico em enfermagem;

- Descrever o número de publicações, a distribuição desses estudos Quanto ao: título defendido, origem da publicação, ano de defesa, metodologia utilizada e temática abordada.

\section{METODOLOGIA}

Este é um estudo bibliográfico, Que se realizou no mês de junho de 2006, por meio de um levantamento da produção científica de enfermagem referente ao ensino clínico no Catálogo de Dissertações e Teses do Centro de Estudos e Pesquisas em Enfermagem (CEPEn) da Associação Brasileira de Enfermagem - ABEn no período de 1979 a $2004^{(7)}$. Para a busca bibliográfica, adotaram-se as seguintes palavras-chave: estágio, ensino clínico, prática em enfermagem e ensino em enfermagem. Os resumos do documento citado foram lidos e deles extraídas informações Quanto à origem da publicação, ano de defesa, metodologia utilizada, descritores e temática abordada.

\section{RESULTADOS}

Com o levantamento realizado do total de 3.093 resumos, encontramos 25 (100\%) artigos relacionados ao objeto da pesQuisa, dos Quais 17 (68\%) são dissertações de mestrado e 8 (32\%) são teses de doutorado. Quanto ao título defendido das produções, houve predomínio de Dissertações de Mestrado em Enfermagem em relação ao Doutorado.

Essas 25 produções distribuem-se da seguinte forma: oito desses trabalhos estudam o processo de ensino-aprendizgem nos estágios, sete fazem uma avaliação dos estágios curriculares, Quatro trabalham com as experiências e percepções vivenciados pelos graduandos no ensino clínico, dois abordam a prática pedagógica do graduando, um identifica incidentes críticos no estágio e um aborda o estágio extracurricular.

A concentração desses estudos foi evidenciada em três estados brasileiros: São Paulo com 15 trabalhos (60\%); Rio de Janeiro, com 5 trabalhos (20\%) e Santa Catarina, com 3 trabalhos (12\%); Minas Gerais, com I trabalho (4\%). Os estados do Ceará e Maranhão apresentaram I único trabalho (4\%), pois tal pesquisa foi realizada por apenas um peseuisador em duas universidades diferente, conforme mostra a Figura 1 .

Analisando a abordagem metodológica utilizada nas 25 


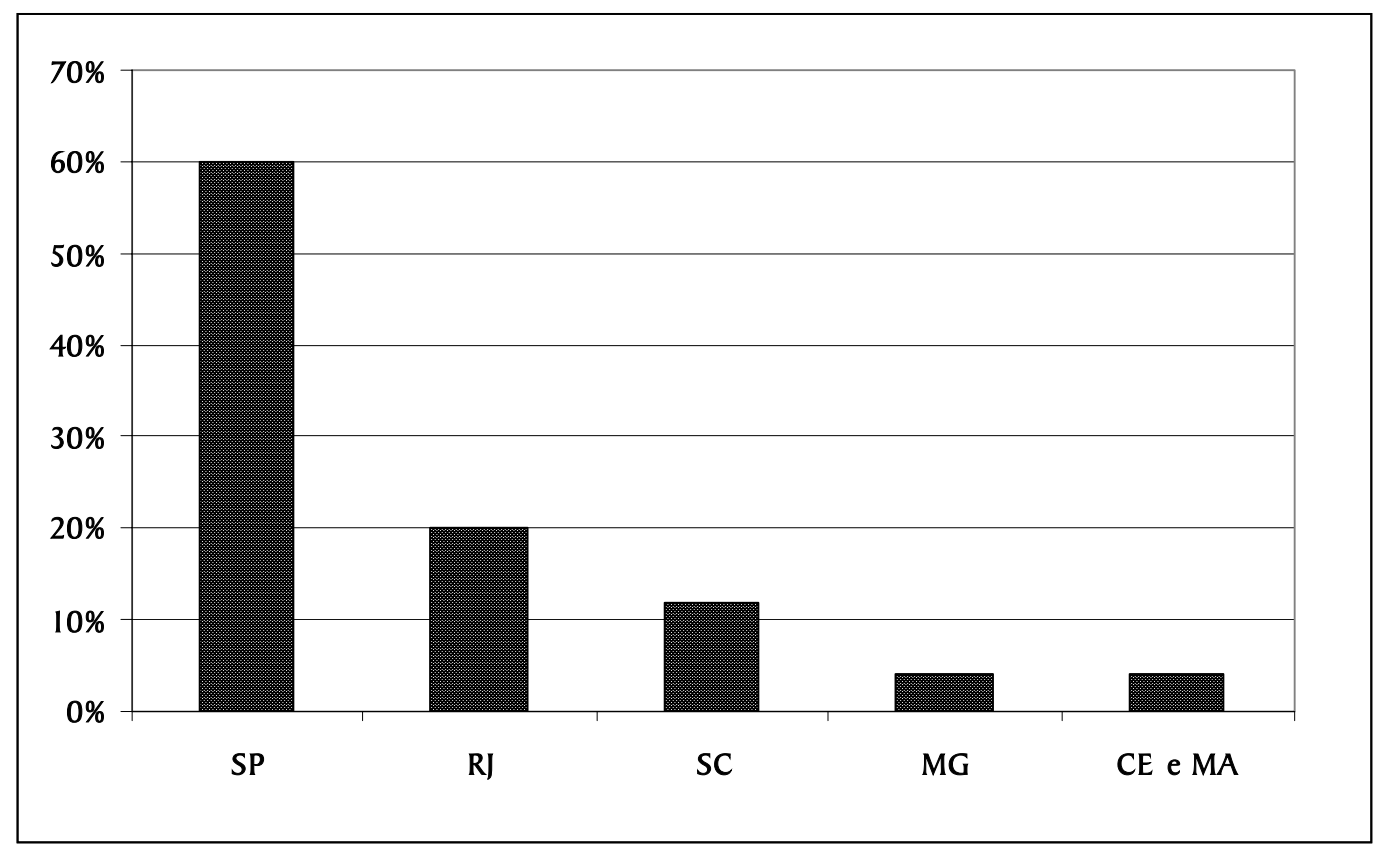

Figura 1. Distribuição da produção científica de enfermagem referente ao ensino clínico por estado brasileiro. São Paulo, 2006.

Tabela 1. Distribuição da produção em enfermagem por ano de realização e por estado brasileiro. São Paulo, 2006.

\begin{tabular}{ccccccc}
\hline Ano de Defesa & SP & R & SC & MG & CE e MA & Total \\
\hline 1972 & 1 & 0 & 0 & 0 & 0 & 1 \\
1973 & 1 & 0 & 0 & 0 & 0 & 1 \\
1989 & 2 & 0 & 0 & 0 & 0 & 2 \\
1990 & 2 & 0 & 0 & 0 & 0 & 2 \\
1991 & 2 & 1 & 0 & 0 & 0 & 3 \\
1992 & 0 & 1 & 0 & 0 & 0 & 1 \\
1993 & 0 & 1 & 1 & 0 & 0 & 2 \\
1996 & 2 & 0 & 0 & 0 & 0 & 2 \\
1997 & 2 & 0 & 0 & 0 & 0 & 2 \\
1999 & 2 & 2 & 2 & 0 & 0 & 6 \\
2001 & 0 & 0 & 0 & 1 & 0 & 1 \\
2002 & 1 & 0 & 0 & 0 & 0 & 1 \\
2003 & 0 & 0 & 0 & 0 & 1 & 1 \\
2004 & 0 & 0 & 0 & 0 & 0 & 0 \\
\hline Total & 15 & 5 & 3 & 1 & 1 & 25 \\
\hline
\end{tabular}

produções pesquisadas, identificamos Que 19, ou seja, 76\% apresentam pesQuisas com abordagem Qualitativa e apenas 6 (24\%) destes estudos utilizaram abordagens Quantitativas.

Outra variável analisada neste estudo foi o ano de publicação dos trabalhos cujos resultados são apresentados na Tabela 1. A compilação desses trabalhos emergentes, segundo o Centro de Estudos e Pesquisas em Enfermagem (CEPEn) ${ }^{(7)}$ da Associação Brasileira de Enfermagem (ABEn) no período de 1979 a 2004 , base de dados do presente estudo, nos mostrou Que no espaço de tempo compreendido entre os anos de 1972 a 1997 houve a produção de um a três trabalhos por ano. Ficou evidente que a maior freQüência da produção científica ocorreu no ano de 1999 , com um total de seis trabalhos. Durante os anos de 2001 a 2004 , verificou-se o menor número de trabalhos, uma produção por ano entre 2001 e 2003, e no ano de 2004 observa-se a inexistência de produção científica nesta área.

\section{DISCUSSÃO}

A produção científica em enfermagem no Brasil é muito recente, principalmente Quando comparada com outras áreas no campo da saúde $^{(8)}$. Esse fato tem se intensificado com a regulamentação da 
pós-graduação "stricto-sensu", e mais recentemente ainda Quando se trata de investigar um fenômeno tão novo como é o ensino, principalmente, Quando se focalizar como objeto de investigação da enfermagem outra categoria como o ensino clínico.

Ao analisar os resumos selecionados na temática em Questão os dados sobre a distribuição da produção científica de enfermagem referente ao ensino clínico de acordo com o título defendido corroboram os informes da CAPES e do Ministério da Educação, Que foram apresentados no $10^{\circ}$ SENADEn (Seminário Nacional de Diretrizes para a Educação em Enfermagem) em 2006. Ele nos mostra Que a maior concentração de programas de pós-graduação (mestrado e doutorado) está na região sudeste e sul, principalmente, no estado de São Paulo(9).

Vale ressaltar Que a preocupação com a educação em enfermagem no Brasil já data desde 1926, ano da criação da Associação Brasileira de Enfermagem (ABEn), por meio das discussões realizadas nos congressos, encontros, oficinas, comissões e das publicações feitas pela Revista Brasileira de Enfermagem. Essa preocupação associada às transformações no campo ideológico, político e econômico do país fez com Que essa entidade firmasse ainda mais o compromisso com a educação de enfermagem, seja ela na graduação, na pós-graduação e no ensino médio.

Esse compromisso ficou ainda mais evidente com a concretização do SENADEn (Seminário Nacional de Diretrizes para a Educação em Enfermagem) em 1994, Que se constitui no fórum de representação da enfermagem brasileira para discussões de assuntos voltados ao ensino, à saúde, à educação e a formação de profissionais de enfermagem. Com isso, acreditamos que os dados apresentados na Tabela I, Que nos mostram um crescimento na produção científica de enfermeiros relacionados ao ensino e formação no campo da enfermagem no final da década de 90 , podem ser justificados pela contribuição importante da ABEn em movimentos envolvidos no currículo e educação em enfermagem ${ }^{(10)}$.

Outro aspecto analisado foi a abordagem metodológica destas produções, Que apresentou predomínio nas pesQuisas Qualitativas. A produção de pesQuisas Qualitativas tem sido um aspecto crescente nos campos de estudo de enfermagem, principalmente Quando estamos discutindo educação e formação em saúde. A focalização de aspectos sociais e culturais Que são característicos dos referenciais das ciências humanas e sociais, contexto em Que está inserido o ensino, não eram, até então, considerados relevantes nas investigações em saúde, campo em Que se insere a enfermagem.

$\mathrm{Na}$ realidade, a criação e a utilização de pesquisas com abordagem metodológica Quantitativa nas diversas áreas do conhecimento estão associadas com a corrente positivista, segundo a Qual, a pesQuisa Qualitativa não fazia muito sentido, por contraporse as idéias na abordagem Quantitativa ${ }^{(11)}$.

\section{CONSIDERAÇÕES FINAIS}

Apesar de o ensino clínico ser considerado como uma etapa muito importante na formação do enfermeiro, já era de se esperar uma incipiente produção científica em enfermagem com abordagem nesse sentido. Neste trabalho foi possível constatar Que há necessidade da realização de mais estudos Que abordem o ensino clínico no campo de enfermagem, pois a prática da assistência de enfermagem é uma atividade muito importante na formação desse profissional. Com a elaboração de estudos voltados para a prática docente, poderemos aperfeiçoar os nossos fazeres encuanto educadores e ocupar de forma competente e Qualificada nosso espaço no sistema de educação e saúde.

Ressaltamos a importância e a necessidade da realização de mais estudos que abordem a formação acadêmica do enfermeiro, pois a movimentação dos saberes teóricos e práticos dos graduandos significa o refinamento de noções e teorias adeuiridas por meio do encontro com várias situações da prática, e o docente envolvido nessas atividades precisa conhecer todas as nuances que permeiam esse processo e com esse conhecimento contribuir no processo de ensino-aprendizagem.

Com isso, os docentes envolvidos no ensino clínico em enfermagem e no processo de formação do enfermeiro devem conhecer os desafios, as possíveis problemáticas e os caminhos existentes que podem auxiliar no sucesso ou fracasso dessa atividade pedagógica.

\section{REFERÊNCIAS}

I. Alarcão I, Rua M. Interdisciplinaridade, estágios clínicos e desenvolvimento de competências. Texto Contexto Enferm 2005; 14(3): 373-82.

2. Carvalho MDB. Expectativas dos alunos de enfermagem frente ao primeiro estágio em hospital. Rev Esc Enferm USP 1999; 33(2): 200-6.

3. Andrade MN. Estágio curricular: avaliação de experiência. Rev Bras Enferm 1989; 42(1): 27-41.

4. Backes VMS. Estilos de pensamentos e práxis na enfermagem. Rio Grande do Sul: Editora Unijui; 2000.

5. Casate IC, Corrêa AK. Vivências de alunos em estágio hospitalar: subsídios para refletir sobre a humanização em saúde. Rev Esc Enferm USP 2006; 40(3):321-8.

6. Silva DM, Silva EMVB. O ensino clínico na formação em enfermagem. Millenium Revista do ISPV [periódico na Internet] 2004 Out; 30(8): 103-18. Disponível em: URL: http:// www.ipv.pt/millenium/Millenium30/8.pdf

7. Associação Brasileira de Enfermagem. Centro de estudos e pesquisas em enfermagem. Informações sobre pesquisas e pesQuisadores em enfermagem. Brasília: ABEn; 1979.

8. Rocha SMM, Silva GB. Linhas filosóficas e ideológicas na pesquisa em enfermagem no Brasil. Rev Bras Enferm 1987; 40(4): 217.

9. Rodriguez RAP, Erdmann AL. Pós-graduação em enfermagem: coneuistas, desafios e perspectivas. CAPES/Ministério da Educação e Cultura. In: Anais do $10^{\circ}$ Seminário Nacional de Diretrizes para a Educação em Enfermagem; 2006 Agosto 912; Brasília (DF), Brasil. Brasília: ABEn; 2006.

10. Mancia JR, Padilha MICS, Reibnitz KS. A contribuição dos SENADEns para a construção das diretrizes curriculares da enfermagem. Anais do $6^{\circ}$ Seminário Nacional de Diretrizes para a Educação em Enfermagem; 2002 Maio 27-30; Teresina (PI), Brasil. Teresina: ABEn-PI; 2002.

1 I. Demo P. Pesquisa Qualitativa: busca de equilíbrio entre forma e conteúdo. Rev Lat-am Enfermagem 1998; 6(2): 89-104. 